\title{
Symptomatic form of muscular dystrophy of Duchenne and Becker in female carriers
}

INSERM

\section{Source}

INSERM. (1999). Orphanet: an online rare disease and orphan drug data base.

Symptomatic form of muscular dystrophy of Duchenne and Becker in female carriers.

ORPHA:206546

Symptomatic forms of Duchenne and Becker muscular dystrophies (DMD and BMD: see these terms) in females carriers are characterized by variable degrees of muscle weakness due to progressive skeletal myopathy, sometimes associated with dilated cardiomyopathy or left ventricle dilation. 